\title{
Volumetric brain morphometry changes in patients with obstructive sleep apnea syndrome: effects of CPAP treatment and literature review
}

\author{
Nelly T. Huynh ${ }^{1,2}$, Olga Prilipko ${ }^{1}$, Clete A. Kushida ${ }^{1}$ and Christian Guilleminault ${ }^{1}{ }^{*}$ \\ 1 Stanford Centre for Sleep Sciences and Medicine, Stanford University, Redwood City, CA, USA \\ 2 Faculty of Dental Medicine, University of Montreal, Montreal, QC, Canada
}

Edited by:

Anne-Marie Landtblom, University of Linköping, Sweden

Reviewed by:

Hiroshi Kadotani, Shiga University of Medical Science, Japan

Isaac Almendros, The University of

Chicago, USA

${ }^{*}$ Correspondence:

Christian Guilleminault, Stanford

Centre for Sleep Sciences and

Medicine, Stanford University, 450

Broadway Street, Redwood City, CA

94063, USA

e-mail:cguil@stanford.edu
Introduction: Obstructive sleep apnea syndrome (OSAS) is a frequent breathing disorder occurring during sleep that is characterized by recurrent hypoxic episodes and sleep fragmentation. It remains unclear whether OSAS leads to structural brain changes, and if so, in which brain regions. Brain region-specific gray and white matter volume (GMV and WMV) changes can be measured with voxel-based morphometry (VBM). The aims of this study were to use VBM to analyze GMV and WMV in untreated OSAS patients compared to healthy controls $(\mathrm{HC})$; examine the impact of OSAS-related variables (nocturnal hypoxemia duration and sleep fragmentation index) on GMV and WMV; and assess the effects of therapeutic vs. sham continuous positive airway pressure (CPAP) treatment. We discuss our results in light of previous findings and provide a comprehensive literature review.

Methods:Twenty-seven treatment-naïve male patients with moderate to severe OSAS and seven healthy age- and education-matched $\mathrm{HC}$ were recruited. After a baseline fMRI scan, patients randomly received either active (therapeutic, $n=14$ ) or sham (subtherapeutic, $n=13$ ) nasal CPAP treatment for 2 months.

Results: Significant negative correlations were observed between nocturnal hypoxemia duration and GMV in bilateral lateral temporal regions. No differences in GMV or WMV were found between OSAS patients and $\mathrm{HC}$, and no differences between CPAP vs. sham CPAP treatment effects in OSAS patients.

Conclusion: It appears that considering VBM GMV changes there is little difference between OSAS patients and HC. The largest VBM study to date indicates structural changes in the lateral aspect of the temporal lobe, which also showed a significant negative correlation with nocturnal hypoxemia duration in our study. This finding suggests an association between the effect of nocturnal hypoxemia and decreased GMV in OSAS patients.

Keywords: obstructive sleep apnea syndrome, volumetric brain morphology, functional magnetic resonance imaging, continuous positive airway pressure, gray matter volume, white matter volume, nocturnal hypoxemia

\section{INTRODUCTION}

Obstructive sleep apnea syndrome (OSAS) is a frequent but insufficiently recognized breathing disorder occurring during sleep that affects at least $2-4 \%$ of the population aged $30-60$ years and up to $20-50 \%$ of the elderly population, with a $2: 1 \mathrm{men} /$ women ratio in Caucasians $(1,2)$. It is characterized by recurrent hypoxic episodes during sleep, sleep fragmentation, and changes in sleep architecture, resulting in increased cardiovascular comorbidity, neurocognitive impairment, and mood disorders, as well as excessive daytime sleepiness (3-8).

Several studies have examined whether OSAS also induces morphological brain changes that could underlie those impairments (9-15). To date, results vary widely across structural neuroimaging studies of OSAS, with both positive and negative results. However, among studies that reported structural changes, hippocampal abnormalities are the most consistent finding across different neuroimaging techniques $(10,11,14,16,17)$.

Voxel-based morphometry (VBM) is a widely used neuroimaging technique that allows non-invasive, region-specific measurement of gray and white matter volume (GMV and WMV) changes in the brain. Previous VBM studies in OSAS patients have reported conflicting results, which cannot be entirely explained by differences in methodologies or disease severity $(9-12,14,18)$. The observation that much of the VBM differences in GMV observed in OSAS patients are reversible using continuous positive airway pressure (CPAP) treatment led to the hypothesis that these decreases reflect either neuronal or vasogenic changes, or both $(14,15)$.

The interest in determining whether OSAS leads to structural brain changes is explained by the fact that some OSAS-related 
deficits are irreversible, which could be related to OSAS-induced cellular loss. Therefore, determining whether OSAS leads to structural brain changes and if so, mapping their anatomical distribution and extent, as well as understanding their nature, will greatly impact OSAS disease prevention, early diagnosis, and management.

Therefore, the aim of this study was to use VBM analysis to (a) compare GMV and WMV in untreated OSAS patients to those in healthy controls (HC); (b) examine the impact of OSAS-related variables (nocturnal hypoxemia duration and sleep fragmentation index) on GMV and WMV; (c) assess the effects of therapeutic vs. sham CPAP treatment; and (d) examine our findings in light of previously published research.

\section{MATERIALS AND METHODS PARTICIPANTS}

Twenty-seven treatment-naïve male patients with moderate to severe OSAS [apnea-hypopnea index $(\mathrm{AHI}) \geq 15$ ] and seven healthy age- and education-matched control subjects $(\mathrm{AHI}<5)$ were recruited from the Stanford Sleep Disorder Clinic through advertisements. All participants were right-handed non-smokers and had regular sleep schedules. Exclusion criteria were other sleep disorders, any neurological or psychiatric disorder, and taking psychotropic medications or medication liable to affect the hemodynamic response (e.g., vasodilators or vasoconstrictors).

After a baseline functional magnetic resonance imaging (fMRI) scan, patients were randomly assigned to either the active (therapeutic, $n=14$ ) or sham (subtherapeutic, $n=13$ ) nasal CPAP group. A CPAP titration study was conducted on all patients in both groups: the active group was effectively titrated to the appropriate nasal CPAP pressure, and the sham group slept with the subtherapeutic nasal CPAP previously used in sham CPAP studies (19). The sham CPAP device closely simulated the active CPAP device with respect to airflow through the exhalation port and operating noise. A prior study using a functionally similar sham CPAP device revealed that oxygen saturation, end-tidal $\mathrm{CO}_{2}$, and mean temperature and humidity measured at the CPAP mask were the same for active and sham CPAP (19), and no significant difference was found in sleep parameters or the number of abnormal respiratory events between the sham CPAP group and a no-treatment group in 10 men with OSAS matched for age and AHI.

Subjects in both groups were treated for 2 months and treatment compliance was monitored using an Encore ${ }^{\circledR}$ Pro SmartCard ${ }^{\circledR}$ system. At the end of the treatment period, the sham CPAP group underwent a second CPAP titration night, were provided with therapeutic CPAP treatment, and left the study.

The study was approved by the Stanford Institutional Review Board (IRB approval No: 5129), and all participants signed an informed consent form. This study was a research fMRI sub-study conducted at one center of a multi-centric clinical trial (APPLES), including five centers and 1200 participants randomized into CPAP and sham CPAP groups (ID No: NCT00051363).

\section{OVERNIGHT SLEEP STUDIES}

Overnight polysomnography (PSG) recordings were performed on all participants at baseline. In OSAS patients, PSG recordings confirmed the moderate to severe OSAS diagnosis $(\mathrm{AHI} \geq 15)$. In healthy volunteers, PSG recordings confirmed the absence of sleep disorders, including OSAS (AHI $<5)$. PSG recordings included electroencephalography, electrooculogram, electrocardiogram, electromyogram for chin and legs, nasal air flow with nasal cannula, abdominal and thoracic respiratory belts, and pulse oximetry. Measures were scored according to AASM criteria (20). PSG recordings were performed using the CleveMed ambulatory Crystal Monitor ${ }^{\circledR}$ or Sandman in-house software.

\section{MAGNETIC RESONANCE IMAGING}

All participants underwent an fMRI scan at baseline and after 2 months of either sham or therapeutic CPAP treatment. Imaging data were obtained with a 3.0-T GE scanner (Milwaukee, WI, USA) using a custom quadrature birdcage head coil. Head movement was minimized with foam padding. High-resolution T1-weighted images $\left(\mathrm{TR}=3000 \mathrm{~ms}, \mathrm{TE}=68 \mathrm{~ms}\right.$, flip angle $=11^{\circ}$, FOV $=25 \mathrm{~cm}$, matrix $=256 \times 256,124$ axial slices, slice thickness $=1.2 \mathrm{~mm}$ ) were collected on each participant using an IR-prep FSPGR sequence for T1 contrast.

\section{VOXEL-BASED MORPHOMETRY AND STATISTICAL ANALYSES}

Imaging data were preprocessed and analyzed using SPM8 software (Wellcome Department of Cognitive Neurology, London, UK) ${ }^{1}$ and the VBM8 Toolbox ${ }^{2}$ with default parameters in MatLab 7.9.0. Preprocessing steps included tissue segmentation, highdimensional DARTEL normalization of modulated data by the non-linear components derived from the normalization matrix (modulated GMV and WMV), and spatial smoothing with a Gaussian kernel of $8 \mathrm{~mm}$ full width at half maximum (FWHM).

Untreated OSAS patients were compared to $\mathrm{HC}$ at baseline on sleep and treatment compliance variables using one-way ANOVA and two-sample $t$-test. Brain morphology was analyzed with a one-way ANOVA for baseline comparisons between the two groups of untreated OSAS patients and HC. Treatment effects were explored with a flexible factorial model in 21 OSAS patients (11 sham CPAP and 10 therapeutic CPAP), as six patients were excluded for low treatment compliance $(<50 \%$ of treatment days at $>4 \mathrm{~h}$ of CPAP use). The effects of sleep fragmentation (measured with $\mathrm{AHI}$ ) and nocturnal hypoxemia duration (in minutes) were assessed by multiple regression analysis in untreated OSAS patients.

\section{RESULTS}

At baseline, untreated OSAS patients were equivalent on all sleep and breathing variables. Patients were then randomly assigned to either treatment group. Both OSAS treatment groups demonstrated similar CPAP compliance (see Table 1).

\section{GRAY MATTER VOLUME}

Using topological false discovery rate (FDR) correction for multiple comparisons at cluster level $(p<0.05$, voxel level $p=0.005$ uncorrected), we found no difference in GMV between OSAS patients and $\mathrm{HC}$ and no effects of CPAP vs. sham CPAP treatment in OSAS patients. In OSAS patients, we found a significant negative correlation between nocturnal hypoxemia duration $\left(<90 \% \mathrm{SaO}_{2}\right)$ and GMV in bilateral lateral temporal regions [middle and inferior temporal gyri: Brodmann Area $(B A)=20,21]$ (see Figure 1).

\footnotetext{
${ }^{1}$ http://www.fil.ion.ucl.ac.uk/spm

${ }^{2}$ http://dbm.neuro.uni-jena.de/vbm/
} 


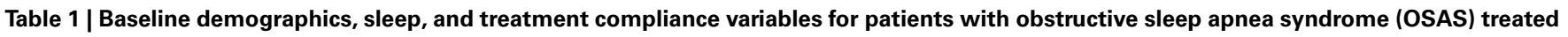
with sham continuous positive airway pressure (CPAP) device or active CPAP device.

\begin{tabular}{|c|c|c|c|c|}
\hline & $\begin{array}{c}\text { Controls } \\
\begin{array}{c}n=7\end{array}\end{array}$ & $\begin{array}{c}\text { Sham CPAP } \\
n=13\end{array}$ & $\begin{array}{c}\text { Active CPAP } \\
n=14\end{array}$ & $p$-Value \\
\hline Age (years) & $41.4 \pm 3.1$ & $44.0 \pm 2.0$ & $42.9 \pm 2.2$ & $0.78^{a} ; 0.48^{b} ; 0.71^{c} ; 0.70^{d}$ \\
\hline BMI $\left(\mathrm{kg} / \mathrm{m}^{2}\right)$ & $24.1 \pm 1.2$ & $26.1 \pm 0.6$ & $28.7 \pm 1.1$ & $\mathbf{0 . 0 1 ^ { \mathbf { a } } ; 0 . 1 2 ^ { b } ; \mathbf { 0 . 0 2 }}{ }^{\mathrm{c}} ; \mathbf{0 . 0 5 ^ { \mathbf { d } }}$ \\
\hline Total sleep time (min) & $349.8 \pm 53.5$ & $376.6 \pm 11.7$ & $378.7 \pm 19.4$ & $0.74^{a} ; 0.66^{b} ; 0.53^{c} ; 0.93^{d}$ \\
\hline Stage 1 TST (\%) & $4.1 \pm 0.7$ & $11.3 \pm 1.8$ & $10.7 \pm 2.2$ & $0.20^{\mathrm{a}} ; \mathbf{0 . 0 4 ^ { \mathbf { b } }} ; \mathbf{0 . 0 \mathbf { 1 } ^ { \mathbf { c } }} ; 0.84^{\mathrm{d}}$ \\
\hline Stage 2 TST (\%) & $61.8 \pm 6.9$ & $61.6 \pm 2.5$ & $66.3 \pm 3.1$ & $0.50^{a} ; 0.97^{b} ; 0.52^{c} ; 0.25^{d}$ \\
\hline Stage 3 TST (\%) & $5.1 \pm 1.8$ & $5.9 \pm 1.5$ & $4.2 \pm 1.2$ & $0.64^{a} ; 0.76^{b} ; 0.73^{c} ; 0.36^{d}$ \\
\hline Stage 4 TST (\%) & $0.7 \pm 0.4$ & $1.5 \pm 0.8$ & $2.1 \pm 1.2$ & $0.75^{a} ; 0.56^{b} ; 0.53^{c} ; 0.68^{d}$ \\
\hline REMTST (\%) & $23.4 \pm 6.1$ & $19.6 \pm 1.1$ & $16.7 \pm 1.5$ & $\mathbf{0 . 0 1 ^ { \mathbf { a } }} ; 0.25^{\mathrm{b}} ; \mathbf{0 . 0 1 ^ { \mathbf { c } }} ; 0.14^{\mathrm{d}}$ \\
\hline $\mathrm{AHI}$ & $2.1 \pm 0.8$ & $34.0 \pm 4.8$ & $43.4 \pm 5.9$ & 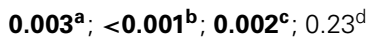 \\
\hline Sleep efficiency (\%) & $84.8 \pm 5.4$ & $83.4 \pm 2.1$ & $82.5 \pm 3.6$ & $0.93^{a} ; 0.78^{b} ; 0.76^{c} ; 0.84^{d}$ \\
\hline Average $\mathrm{SPO}_{2}(\%)$ total & $104.7 \pm 7.5$ & $95.4 \pm 0.3$ & $93.7 \pm 1.0$ & $\mathbf{0 . 0 1 ^ { \mathbf { a } }} ; 0.30^{\mathrm{b}} ; 0.24^{\mathrm{c}} ; 0.11^{\mathrm{d}}$ \\
\hline Number days range & N/A & $72.1 \pm 5.1$ & $78.3 \pm 5.7$ & $0.43^{d}$ \\
\hline Average usage - all days (h/night) & N/A & $4.7 \pm 0.5$ & $4.4 \pm 0.4$ & $0.57^{d}$ \\
\hline Percent days $>4 \mathrm{~h}-$ all days & N/A & $67.1 \pm 8.5$ & $62.0 \pm 6.9$ & $0.64^{d}$ \\
\hline
\end{tabular}

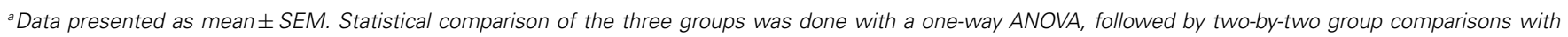
independent t-test.

${ }^{b}$ Control vs. sham.

${ }^{c}$ Control vs. active.

${ }^{a}$ Sham vs. active.

N/A, not applicable.
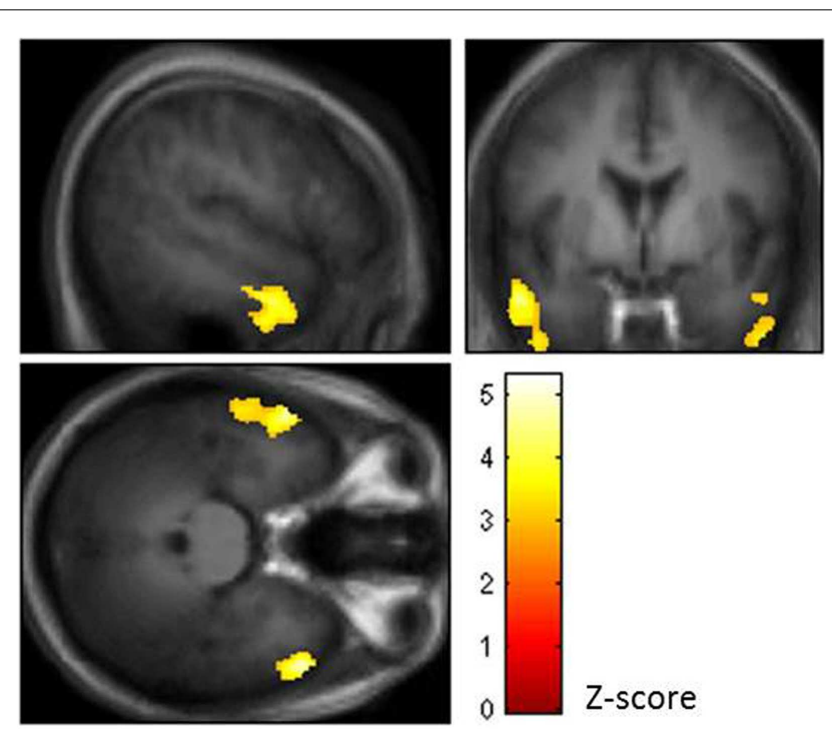

Z-score

FIGURE 1 | Brain regions showing negative correlation between nocturnal hypoxemia duration and gray matter volume (GMV) in bilateral lateral temporal regions of the middle and inferior temporal gyri $(B A=20,21)$ [topological false discovery rate (FDR) correction for multiple comparisons at cluster level $p<0.05$, voxel level $p=0.005$ uncorrected].

Using a voxel-wise $p<0.001$ threshold uncorrected for multiple comparisons with a 20-voxel extent threshold for exploratory analysis, we found significantly higher GMV in HC compared to
OSAS patients in the left middle frontal gyrus $(B A=46)$. Significantly higher GMV was found in untreated OSAS patients compared to $\mathrm{HC}$ in the right precuneus $(\mathrm{BA}=7)$ and fusiform/lingual gyri $(\mathrm{BA}=18)$ (see Table 2$)$.

A significant positive correlation was found between GMV and $\mathrm{AHI}$ in the left inferior parietal lobule $(\mathrm{BA}=40)$. Significant negative correlations were found between GMV and $\mathrm{AHI}$ in the right uncus $(B A=36)$, left gyrus rectus $(B A=11)$, and left precuneus $(\mathrm{BA}=7)$ (see Table 2).

Four OSAS patients were excluded from the multiple comparison analysis due to incomplete nocturnal saturation data. A significant positive correlation was found in the left precuneus $(\mathrm{BA}=7)$. Significant negative correlations between GMV and nocturnal hypoxemia duration were found in the left middle temporal gyri $(\mathrm{BA}=21)$, right superior temporal gyrus $(\mathrm{BA}=38)$, left inferior temporal gyrus $(B A=20)$, and uncus $(B A=36)$ (see Table 2).

No regions showed a significant group $\times$ session interaction. Furthermore, no correlations were found between CPAP compliance and GMV in bilateral hippocampal regions of interest (ROIs), and no negative correlations with nocturnal hypoxemia.

\section{WHITE MATTER VOLUME}

Using a voxel-wise $p<0.001$ threshold uncorrected for multiple comparisons with a 20-voxel extent threshold for exploratory analysis, we found significantly higher WMV in HC compared to OSAS patients in the right occipital lingual gyrus and the left middle occipital gyrus (see Table 3). Similarly to the GMV analyses, four OSAS patients were excluded from the multiple comparison analysis due to incomplete nocturnal saturation data. Significant 
Table 2 | Gray matter volume (GMV) analysis results: (A) brain regions showing significant differences in GMV between healthy controls (HC) and obstructive sleep apnea syndrome (OSAS) patients; (B) brain regions showing significant correlation with the apnea-hypopnea index (AHI) or with nocturnal hypoxemia duration in OSAS patients (MNI coord.; 20 vox., uncorrected $p<0.001$ ).

\begin{tabular}{|c|c|c|c|c|c|c|c|}
\hline & Region & Brodmann area & $\boldsymbol{X}$ & $Y$ & $Z$ & $Z$-score & Cluster size \\
\hline \multicolumn{8}{|c|}{ (A) COMPARISON BETWEEN HC AND OSAS PATIENTS } \\
\hline $\mathrm{HC}>\mathrm{OSAS}$ & $\mathrm{L}$ middle frontal gyrus & 46 & -43 & 44 & 9 & 3.31 & 52 \\
\hline & R occipital fusiform gyrus & 18 & 26 & -87 & -19 & 3.56 & 69 \\
\hline & R occipital lingual gyrus & 18 & 2 & -86 & -7 & 3.41 & 123 \\
\hline \multirow[t]{3}{*}{ AHI negative } & $L$ medial frontal gyrus & 11 & -1 & 48 & -17 & 4.07 & 229 \\
\hline & $R$ uncus & 36 & 21 & 1 & -35 & 3.80 & 187 \\
\hline & $\mathrm{R}$ precuneus & 7 & 18 & -71 & 44 & 3.78 & 97 \\
\hline Desaturation positive & $L$ precuneus & 7 & -17 & -60 & 44 & 4.70 & 96 \\
\hline Desaturation negative & $\mathrm{R}$ superior temporal gyrus & 38 & 46 & 15 & -33 & 4.17 & 602 \\
\hline
\end{tabular}

Table 3 | White matter volume (WMV) analysis results: (A) brain regions showing significant differences in GMV between healthy controls (HC) and obstructive sleep apnea syndrome (OSAS) patients; (B) brain regions showing significant correlation with nocturnal hypoxemia duration in OSAS patients (MNI coord.; 20 vox., uncorrected $p<0.001$ ).

\begin{tabular}{|c|c|c|c|c|c|c|}
\hline & Region & $x$ & $Y$ & $z$ & $Z$-score & Cluster size \\
\hline \multicolumn{7}{|c|}{ (A) COMPARISON BETWEEN HC AND OSAS PATIENTS } \\
\hline \multirow[t]{2}{*}{$\mathrm{HC}>\mathrm{OSAS}$} & $\begin{array}{l}\text { R occipital } \\
\text { lingual gyrus }\end{array}$ & 20 & -50 & -3 & 3.87 & 351 \\
\hline & $\begin{array}{l}\text { L middle } \\
\text { occipital gyrus }\end{array}$ & -23 & -85 & 15 & 3.54 & 23 \\
\hline \multicolumn{7}{|c|}{ (B) CORRELATIONS IN OSAS PATIENTS } \\
\hline \multirow[t]{2}{*}{$\begin{array}{l}\text { Desaturation } \\
\text { positive }\end{array}$} & $\begin{array}{l}\mathrm{L} \text { medial } \\
\text { frontal gyrus }\end{array}$ & -7 & 1 & 48 & 3.88 & 69 \\
\hline & R frontal & 20 & -10 & 52 & 3.87 & 64 \\
\hline
\end{tabular}

positive correlations between WMV and nocturnal hypoxemia duration were found in the left medial frontal gyrus and the right frontal gyrus (see Table 3).

\section{DISCUSSION}

Voxel-based morphometry (VBM) allows voxel-wise comparisons of brain structures by performing statistical analyses of MR images that have undergone standard preprocessing, including gray matter (GM) and white matter (WM) segmentation, normalization to an MNI anatomical template, and spatial smoothing. However, normalization introduces a distortion in the local MR signal due to individual structural variability, resulting in overestimation of a given structure's signal if it has been stretched, or underestimation if it has been reduced to fit the standard anatomical template. To circumvent this issue, Good et al. (21) proposed an additional data treatment step in which GM voxel values are multiplied by Jacobian determinants (representing deformation parameters obtained after normalization), resulting in modulated data. Modulated (GM or WM volume) and unmodulated (GMor WM concentration) data yield different information about examined brain structures. Modulated GM or WM volume (GMV or WMV) images allow comparing absolute brain volume, thus inferring the presence of atrophy in a given brain structure, whereas unmodulated GM or WM concentration (GMC or WMC) images allow comparing the ratio of GM or WM to other brain tissue present in a given brain structure (22). However, unmodulated images do not allow inferences about structural volume differences between brain structures indifferent subjects or groups of subjects. Moreover, GMC can be misinterpreted as meaning the concentration of neurons in a given voxel, leading to erroneous interpretations of potential cellular injury. Therefore, to respond to the question of whether the brain of OSAS patients presents global or focal parenchymal atrophy compared to the brain of $\mathrm{HC}$, analyzing GMV and WMV appears more appropriate than analyzing GMC and WMC.

Although VBM studies of OSAS patient brains have obtained highly inconsistent results, GMV analyses show few or no differences between patients and HC. Moreover, the majority of studies reporting GMV differences present results that are uncorrected for multiple comparisons, suggesting that the observed effects are of small magnitude (see Table 4).

The first VBM-based study in OSAS patients was published by Macey et al. (10) (1.5 T) in 21 patients with a mean respiratory disturbance index (RDI) of $38 \pm 24$ events/h. The analysis was performed on unmodulated data (GMC), and patients were not screened for cardiovascular or psychiatric comorbidities. Results were reported for $p=0.001$ uncorrected for multiple comparisons at a 350-voxel extent.

Higher GM signals were reported in OSAS patients compared to $\mathrm{HC}$ in the right postcentral gyrus, posterior lateral parietal and anterior superior frontal cortex bilaterally, and bilateral parahippocampal gyri, among others. No regions showed higher GM 


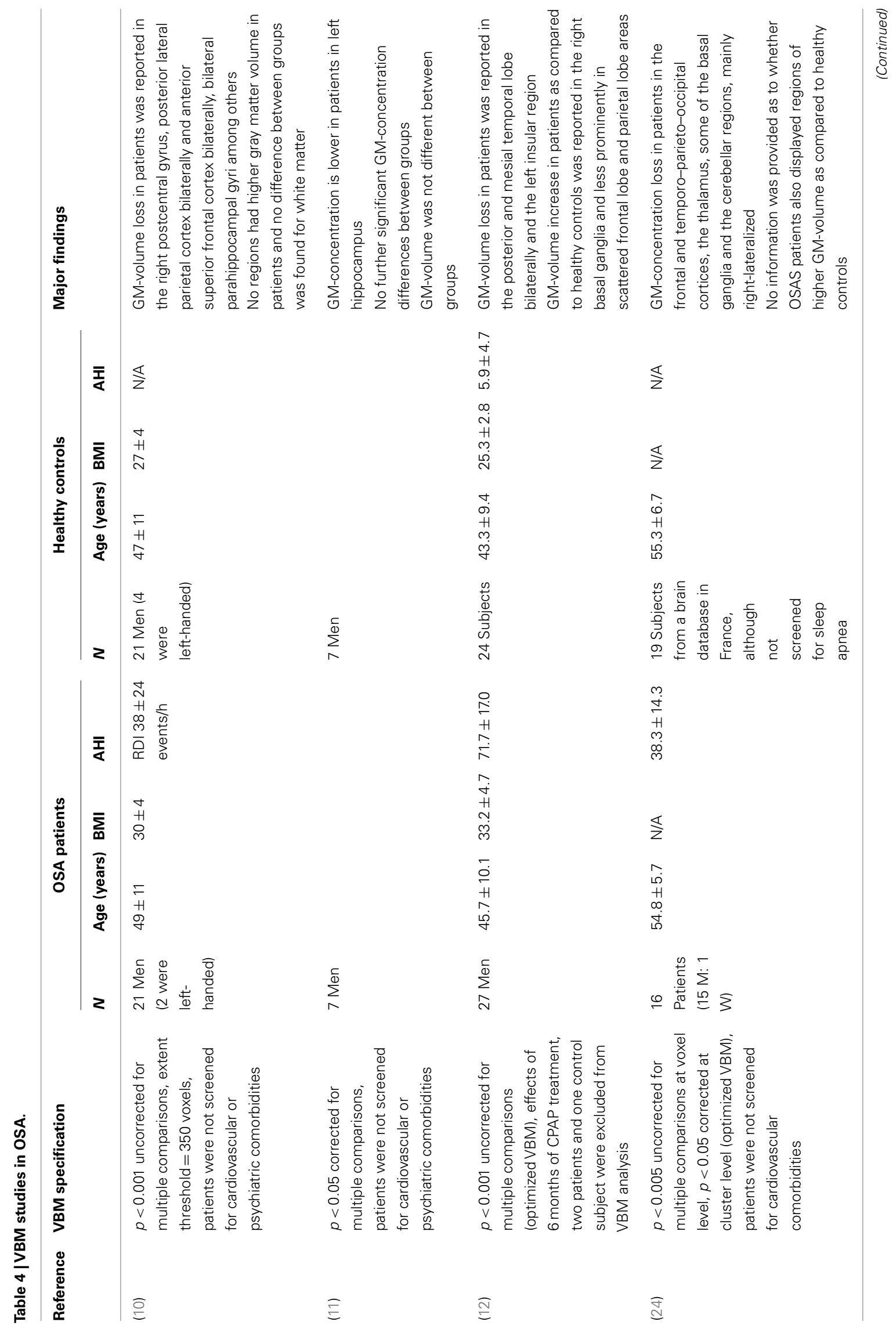




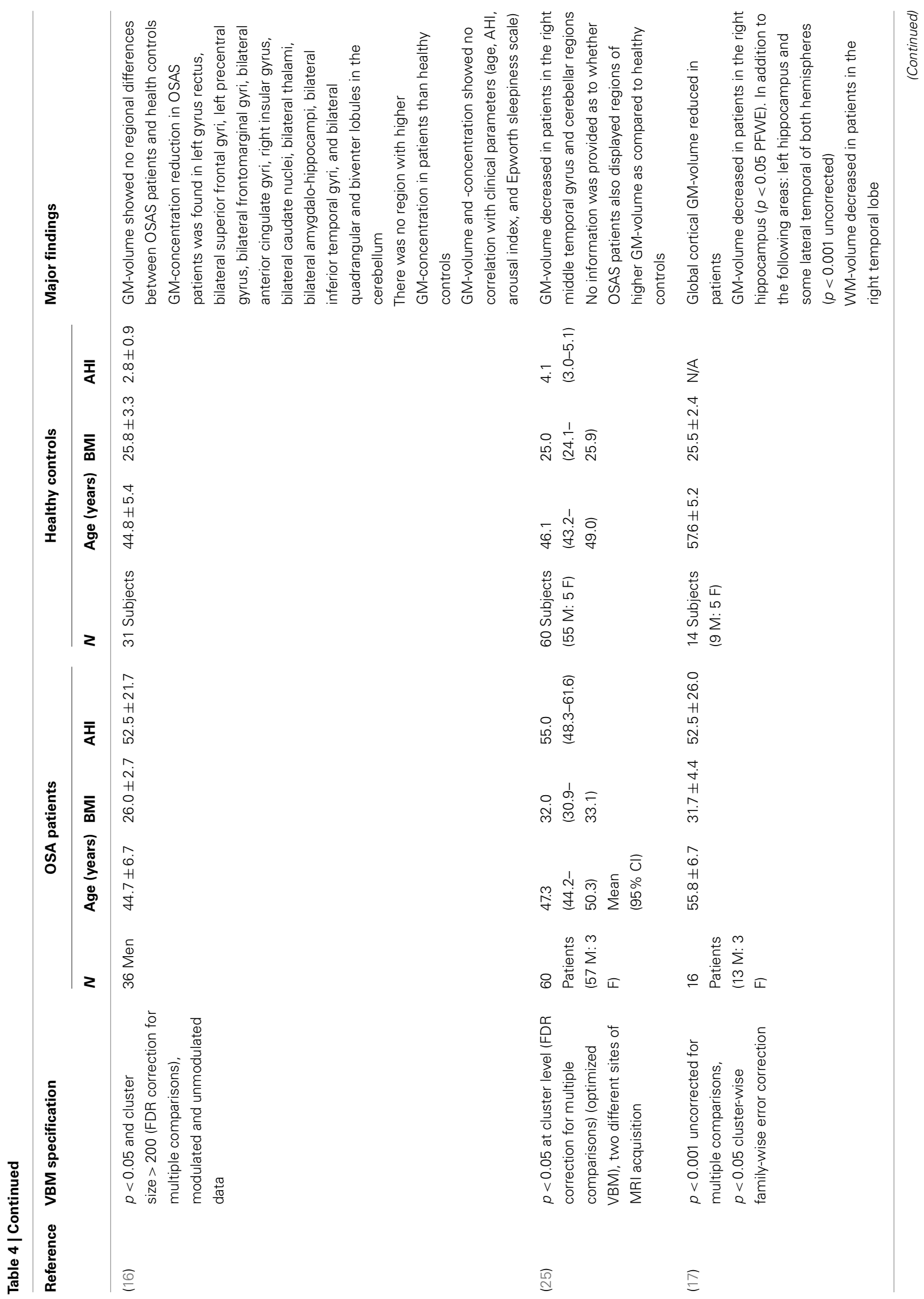




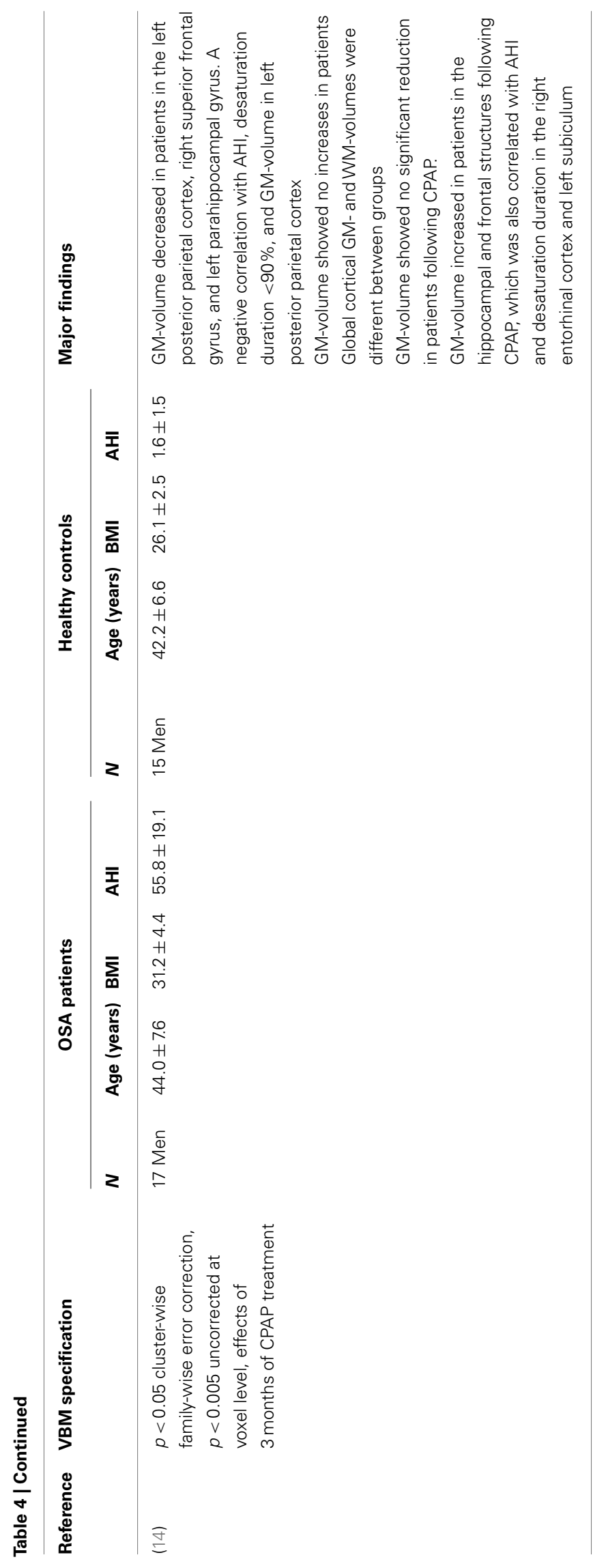

signals in OSAS patients compared to HC, and no differences in WM were found.

Morrell et al. (11) (1.5 T) also examined unmodulated data (GMC) in seven male OSAS patients with a mean AHI of 28 events/h and found lower GMC in OSAS patients compared to HC in the left hippocampal region of interest $(p=0.004$, corrected for multiple comparisons, small volume correction). They found no differences between HC and patients on whole brain comparison, $p=0.05$ corrected for multiple comparisons. The same research group found similar findings in 22 patients in another study, with decreased GMC in bilateral posterior hippocampal regions $(p=0.001$, ROI analysis, uncorrected for multiple comparisons) (23).

The first study to use modulated VBM data (GMV) and to investigate the effects of 6 months of CPAP treatment was performed by O'Donoghue et al. (12) (3 T) in 27 patients with severe OSAS (AHI: 74 events/h) without comorbidities. Using a $p$ threshold of 0.001 uncorrected for multiple comparisons, they observed scattered areas of GM deficit in patients compared to control subjects, including the posterior and mesial temporal lobe bilaterally and the left insular region. A reverse contrast showed increased GMV in OSAS patients compared to HC in the right basal ganglia, and less prominently in scattered frontal lobe and parietal lobe areas. However, using multiple comparison correction, they found no differences between OSAS patients and $\mathrm{HC}$, and no changes in OSAS patients after 6 months of CPAP treatment.

Yaouhi et al. (24) (1.5 T) examined brain structure in 16 newly diagnosed OSAS patients (AHI: 38 events/h) using both PET and VBM (unmodulated VBM data). Voxel-wise GMC analysis at $p=0.005$ uncorrected for multiple comparisons ( $p=0.05$ corrected at cluster level) revealed scattered sites of GMC loss in OSAS patients in the frontal and temporo-parieto-occipital cortices, the thalamus, some of the basal ganglia, and the cerebellar regions. No results were provided on the opposite contrast, that is, whether OSAS patients also showed regions of higher GMC compared to HC. Macey et al. (10) found similar results in subjects with cardiovascular comorbidities.

A study by Joo et al. (16) (1.5 T) examined both modulated and unmodulated data from 36 male OSAS patients without cardiovascular, neurological, or psychiatric comorbidities (AHI: 52.5 events/h). Using FDR correction for multiple comparisons $(p=0.05$ and cluster size $>200)$, they found no differences in GMV between OSAS patients and HC, and no brain regions with significant correlations between clinical parameters (age, AHI, arousal index, and Epworth Sleepiness Scale) and GMV. However, GMC was significantly reduced in OSAS patients compared to HC in the bilateral superior frontal gyri, left gyrus rectus, bilateral frontomarginal gyri, bilateral anterior cingulate gyri, right anterior insular gyrus, bilateral caudate nuclei, bilateral thalami, bilateral amygdalohippocampal gyri, bilateral inferior temporal gyri, and bilateral cerebellar cortices. No region showed higher GMC in patients compared to HC.

Canessa et al. (14) (3 T) conducted another VBM study to examine the effects of CPAP treatment on GMV in OSAS patients compared to HC. They studied 17 treatment-naïve OSAS patients (AHI: 55.8 events/h) without associated comorbidities. Using a family-wise error (FWE) cluster-level correction for multiple 
comparisons ( $p=0.05$, voxel level $p=0.005)$, they found significantly reduced GMV in pretreatment patients compared with control subjects in the left posterior parietal cortex and right superior frontal gyrus. No region showed higher GMV in patients with OSAS compared to HC. A significant negative correlation was found between $\mathrm{AHI}$ and time of nocturnal desaturation below $90 \%$ $\mathrm{SaO}_{2}$ and GMV in the left posterior parietal cortex. Furthermore, specifically in the hippocampal region of interest with an uncorrected $p=0.005$ at voxel level, they found decreased GMV in OSAS patients compared to HC. After 3 months of CPAP treatment, specific hippocampal (left subiculum and bilateral entorhinal cortex) and frontal (superior and middle frontal gyri and medial orbitofrontal cortex) clusters showed increased GMV (FDR corrected at cluster level, $p=0.05$ ). Moreover, after CPAP treatment, overall GMV was significantly increased in patients with OSAS, despite no significant increase in total intracranial volume.

The largest VBM study to date, recently published by Morrell et al. (25), investigated GMV in 60 OSAS patients (AHI: 50 events/h) without comorbidities examined at two different sites $(1.5$ and $3 \mathrm{~T})$. Using topological FDR correction for multiple comparisons ( $p=0.05$, cluster level), they found lower GMV in the right middle temporal gyrus and cerebellar regions in patients with OSAS compared to HC. No information was provided as to whether results were provided on higher GMC in OSAS patients also displayed regions of higher GM-concentration compared to HC.

Torelli et al. (17) examined GMV in 16 newly diagnosed OSAS patients (AHI: 63 events/h) without major cardiovascular disorders $(3 \mathrm{~T})$. They found reduced cortical GMV in OSAS patients compared to controls ( $p=0.01$, multiple comparison correction data not provided), as well as a region of decreased GMV in the right hippocampus when applying cluster-wise FWE correction for multiple comparisons $(p=0.05)$.

The above studies indicate that decreased GMV in OSAS patients compared to $\mathrm{HC}$, when applying a statistical correction for multiple comparisons, were found only in the right temporal cortex (25) and the left posterior parietal and right superior frontal corci (14). Increased GMV after 3 months of CPAP treatment were found in the hippocampal and frontal regions, differing from brain regions that showed decreased GMV compared to HC at baseline (14).

The results of our study are in line with previous observations, as we found no GMV or WMV differences between 27 treatment-naïve OSAS patients and $\mathrm{HC}$ when data were corrected for multiple comparison. Using a more lenient threshold (voxelwise $p=0.001$ uncorrected, 20-voxel extent threshold), we found small GMV decreases in the left frontal cortex, but also increases in the right parietal and occipital regions. Given that our subjects had less severe disease (AHI, nocturnal hypoxemia) than subjects in previous studies, our results extend previous observations to a more moderate range of OSAS and support the finding that GMV changes are not prevalent in the brain of OSAS patients.

Interestingly, compared to the findings of Morrell et al., we found a significant negative correlation $(p=0.05$, FDR corrected at cluster level) between nocturnal hypoxemia duration and GMV in the same region of the temporal lobe as their finding of decreased GMV in OSAS patients compared to HC. This suggests an association between the effect of nocturnal hypoxemia and decreased GMV in OSAS patients compared to HC. We did not expect the lateral temporal regions to show a negative correlation with hypoxemia, whereas the hippocampal regions did not. However, as Morrell et al. noted, this area is susceptible to hypoxic damage in an animal model of OSAS (25). Neurons in the dentate gyrus of the hippocampal formation have been shown to maintain their ability to regenerate throughout adult life, and this neurogenesis can be inhibited by sleep fragmentation or deprivation (26). The fact that hippocampal neurons can regenerate even though they are sensitive to oxidative injury, but that the neurogenesis can be inhibited by insufficient sleep, could explain the inconsistent VBM findings in the hippocampus of OSAS patients.

The fact that we found no difference between patients and controls, or between treatment groups while finding a significant correlation with nocturnal hypoxemia in the temporal lobe GMV may appear contradictory, but in reality reflects two different aspects of OSAS effects. On one hand, it indicates that OSAS-related morphological changes, if present, are of small magnitude. On the other, it demonstrates that between AHI and nocturnal hypoxemia duration, the latter is the OSAS-related factor that has the most consistent effect on GMV, particularly in the temporal lobes. Lateral temporal regions that showed correlation with hypoxemia in our study and differences in GMV in Morrell et al.'s work (11, $23,25)$ are involved in various associative processes, particularly memory processing. However, in order to better understand the relationship between these cortical areas and cognitive deficits in OSAS patients, further larger patient sample studies that simultaneously measure both GMV and cognitive impairment are needed. Moreover, more severe hypoxemia is often seen in overweight or obese patients with OSAS. A larger group of patients would allow investigation of findings in normal weight and overweight OSAS patients and of the role of obesity by dissociating OSAS from obesity.

It is unclear why disease severity appears to correlate poorly with GMV changes in OSAS patients. One possible explanation is that factors other than disease severity or vascular comorbidity could be at play. In particular, Alchanatis et al. (27) showed that high intelligence (measured by IQ) has a protective effect on the cognitive function of patients with OSAS. No VBM studies to date have included a measure of education level (which is a surrogate for many personal, socioeconomic, and cognitive variables) in their analysis, and further studies are needed to determine whether patients' education level can explain the discrepancies observed in structural brain studies.

Another question that remains unanswered is which pathophysiological processes are represented by changes in VBM, GM, or WM signals. It has been postulated that decreased GMV represents cellular loss, as seen in local and diffuse brain atrophy. However, in view of the fact that both decreased and increased signals have been observed in OSAS patients, Canessa et al. (14) proposed that changes in VBM signals may be less specific, and may represent neuronal or vascular processes, or both. Future studies could aim to elucidate this question by combining VBM with perfusion and water-sensitive imaging. 
In conclusion, in terms of VBM changes in GMV, there is little difference between OSAS patients and HC. The largest VBM study to date points to structural changes in the lateral aspect of the temporal lobe, which also demonstrated a significant negative correlation with nocturnal hypoxemia duration in our study. Further research is needed to elucidate the potential protective role of cognitive reserve and to distinguish the neuronal and vascular glial contributions to VBM-measured GMV signal changes.

\section{ACKNOWLEDGMENTS}

The authors would like to thank Pierre Rompré, M.Sc. and Nadia Gosselin, Ph.D. for their help and support with data analyses. Equipment and testing was supported by NHLBI through APPLES (5UO1-HL-068060) and Philips Respironics. This study was conducted under post-doctoral fellowships granted to Drs. N. Huynh (Sleep Disorders Foundation) and O. Prilipko (Swiss National Foundation for Scientific Research and FSBMB).

\section{REFERENCES}

1. Young T, Palta M, Dempsey J, Skatrud J, Weber S, Badr S. The occurrence of sleep-disordered breathing among middle-aged adults. N Engl J Med (1993) 328:1230-5. doi:10.1056/NEJM199304293281704

2. Ancoli-Israel S, Alessi C. Sleep and aging. Am J Geriatr Psychiatry (2005) 13:341-3. doi:10.1176/appi.ajgp.13.5.341

3. Bedard MA, Montplaisir J, Richer F, Rouleau I, Malo J. Obstructive sleep apnea syndrome: pathogenesis of neuropsychological deficits. J Clin Exp Neuropsychol (1991) 13:950-64. doi:10.1080/01688639108405110

4. Parish JM, Somers VK. Obstructive sleep apnea and cardiovascular disease. Mayo Clin Proc (2004) 79:1036-46. doi:10.4065/79.8.1036

5. Yaggi H, Mohsenin V. Obstructive sleep apnea and stroke. Lancet Neurol (2004) 3:333-42. doi:10.1016/S1474-4422(04)00766-5

6. Caples SM, Gami AS, Somers VK. Obstructive sleep apnea. Ann Intern Med (2005) 142:187-97. doi:10.7326/0003-4819-142-3-200502010-00010

7. Wolk R, Gami AS, Garcia-Touchard A, Somers VK. Sleep and cardiovascular disease. Curr Probl Cardiol (2005) 30:625-62. doi:10.1016/j.cpcardiol.2005.07.002

8. Coccagna G, Pollini A, Provini F. Cardiovascular disorders and obstructive sleep apnea syndrome. Clin Exp Hypertens (2006) 28:217-24. doi:10.1080/ 10641960600549090

9. Kamba M, Inoue Y, Higami S, Suto Y, Ogawa T, Chen W. Cerebral metabolic impairment in patients with obstructive sleep apnea: an independent association of obstructive sleep apnea with white matter change. J Neurol Neurosurg Psychiatry (2001) 71:334-9. doi:10.1136/jnnp.71.3.334

10. Macey PM, Henderson LA, Macey KE, Alger JR, Frysinger RC, Woo MA, et al. Brain morphology associated with obstructive sleep apnea. Am J Respir Crit Care Med (2002) 166:1382-7. doi:10.1164/rccm.200201-050OC

11. Morrell MJ, McRobbie DW, Quest RA, Cummin AR, Ghiassi R, Corfield DR. Changes in brain morphology associated with obstructive sleep apnea. Sleep Med (2003) 4:451-4. doi:10.1016/S1389-9457(03)00159-X

12. O’Donoghue FJ, Briellmann RS, Rochford PD, Abbott DF, Pell GS, Chan CH, et al. Cerebral structural changes in severe obstructive sleep apnea. Am J Respir Crit Care Med (2005) 171:1185-90. doi:10.1164/rccm.200406-738OC

13. Macey PM, Kumar R, Woo MA, Valladares EM, Yan-Go FL, Harper RM. Brain structural changes in obstructive sleep apnea. Sleep (2008) 31:967-77.

14. Canessa N, Castronovo V, Cappa SF, Aloia MS, Marelli S, Falini A, et al. Obstructive sleep apnea: brain structural changes and neurocognitive function before and after treatment. Am J Respir Crit Care Med (2011) 183:1419-26. doi:10.1164/rccm.201005-0693OC
15. O'Donoghue FJ, Wellard RM, Rochford PD, Dawson A, Barnes M, Ruehland WR, et al. Magnetic resonance spectroscopy and neurocognitive dysfunction in obstructive sleep apnea before and after CPAP treatment. Sleep (2012) 35:41-8. doi:10.5665/sleep. 1582

16. Joo EY, Tae WS, Lee MJ, Kang JW, Park HS, Lee JY, et al. Reduced brain gray matter concentration in patients with obstructive sleep apnea syndrome. Sleep (2010) 33:235-41.

17. Torelli F, Moscufo N, Garreffa G, Placidi F, Romigi A, Zannino S, et al. Cognitive profile and brain morphological changes in obstructive sleep apnea. Neuroimage (2011) 54:787-93. doi:10.1016/j.neuroimage.2010.09.065

18. Macey PM, Kumar R, Yan-Go FL, Woo MA, Harper RM. Sex differences in white matter alterations accompanying obstructive sleep apnea. Sleep (2012) 35:1603-13. doi:10.5665/sleep.2228

19. Farre R, Hernandez L, Montserrat JM, Rotger M, Ballester E, Navajas D. Sham continuous positive airway pressure for placebo-controlled studies in sleep apnea. Lancet (1999) 353:1154. doi:10.1016/S0140-6736(99)01056-9

20. AASM. The AASM Manual for the Scoring of Sleep and Associated Events: Rules, Terminology and Technical Specifications. Westchester, IL: American Academy of Sleep Medicine (AASM) (2007).

21. Good CD, Johnsrude IS, Ashburner J, Henson RN, Friston KJ, Frackowiak RS. A voxel-based morphometric study of ageing in 465 normal adult human brains. Neuroimage (2001) 14:21-36. doi:10.1006/nimg.2001.0786

22. Mechelli A, Price CJ, Friston KJ, Ashburner J. Voxel-based morphometry of the human brain: methods and applications. Curr Med Imaging Rev (2005) 1:105-13. doi:10.2174/1573405054038726

23. Morrell MJ, Twigg G. Neural consequences of sleep disordered breathing: the role of intermittent hypoxia. Adv Exp Med Biol (2006) 588:75-88. doi:10.1007/ 978-0-387-34817-9_8

24. Yaouhi K, Bertran F, Clochon P, Mezenge F, Denise P, Foret J, et al. A combined neuropsychological and brain imaging study of obstructive sleep apnea. J Sleep Res (2009) 18:36-48. doi:10.1111/j.1365-2869.2008.00705.x

25. Morrell MJ, Jackson ML, Twigg GL, Ghiassi R, McRobbie DW, Quest RA, et al. Changes in brain morphology in patients with obstructive sleep apnea. Thorax (2010) 65:908-14. doi:10.1136/thx.2009.126730

26. Meerlo P, Mistlberger RE, Jacobs BL, Heller HC, McGinty D. New neurons in the adult brain: the role of sleep and consequences of sleep loss. Sleep Med Rev (2009) 13:187-94. doi:10.1016/j.smrv.2008.07.004

27. Alchanatis M, Zias N, Deligiorgis N, Amfilochiou A, Dionellis G, Orphanidou D. Sleep apnea-related cognitive deficits and intelligence: an implication of cognitive reserve theory. J Sleep Res (2005) 14:69-75. doi:10.1111/j.1365-2869.2004. 00436.x

Conflict of Interest Statement: The authors declare that the research was conducted in the absence of any commercial or financial relationships that could be construed as a potential conflict of interest.

Received: 17 January 2014; accepted: 07 April 2014; published online: 29 April 2014. Citation: Huynh NT, Prilipko O, Kushida CA and Guilleminault C (2014) Volumetric brain morphometry changes in patients with obstructive sleep apnea syndrome: effects of CPAP treatment and literature review. Front. Neurol. 5:58. doi: 10.3389/fneur.2014.00058

This article was submitted to Sleep and Chronobiology, a section of the journal Frontiers in Neurology.

Copyright (ङ) 2014 Huynh, Prilipko, Kushida and Guilleminault. This is an open-access article distributed under the terms of the Creative Commons Attribution License (CC $B Y)$. The use, distribution or reproduction in other forums is permitted, provided the original author(s) or licensor are credited and that the original publication in this journal is cited, in accordance with accepted academic practice. No use, distribution or reproduction is permitted which does not comply with these terms. 